Meta

Journal des traducteurs

Translators' Journal

\title{
Lexique commun et lexique de métier
}

Table, Rabot, Bouvet

\section{Michel Dessaint}

Volume 39, numéro 4, décembre 1994

Hommage à Bernard Quemada : termes et textes

URI : https://id.erudit.org/iderudit/002559ar

DOI : https://doi.org/10.7202/002559ar

Aller au sommaire du numéro

Éditeur(s)

Les Presses de l'Université de Montréal

ISSN

0026-0452 (imprimé)

1492-1421 (numérique)

Découvrir la revue

Citer cet article

Dessaint, M. (1994). Lexique commun et lexique de métier : table, Rabot, Bouvet. Meta, 39(4), 731-735. https://doi.org/10.7202/002559ar
Résumé de l'article

La qualité comme la continuité de la documentation, pour ce qui est de la France, permettent de suivre l'évolution du lexique des métiers du bois depuis le XVII ${ }^{\mathrm{e}}$ siècle jusqu'au XX ${ }^{\mathrm{e}}$, où l'utilisation de plus en plus exclusive des machines modifie du tout au tout technique et nomenclature. Nous voudrions, par rapport à la langue et au sens commun, situer le vocabulaire de ces métiers, dont les praticiens sont très proches de la vie quotidienne. Aujourd'hui la langue des anciens métiers du bois abandonne peu à peu la langue commune, juste après le moment où elle s'était constituée en langue de spécialité bien répertoriée.
Tous droits réservés @ Les Presses de l'Université de Montréal, 1994
Ce document est protégé par la loi sur le droit d'auteur. L'utilisation des services d'Érudit (y compris la reproduction) est assujettie à sa politique d'utilisation que vous pouvez consulter en ligne.

https://apropos.erudit.org/fr/usagers/politique-dutilisation/ 


\title{
LEXIQUE COMMUN ET \\ LEXIQUE DE METIER \\ TABLE, RABOT, BOUVET
}

MiCHEL DESSAINT

Université Paris $I V$ - Sorbonne, Paris, France

\begin{abstract}
Résumé
La qualité comme la continuité de la documentation, pour ce qui est de la France. permettent de suivre l'évolution du lexique des métiers du bois depuis le XVIIe siecle jusqu'au XX $X^{e}$, où l'utilisation de plus en plus exclusive des machines modifie du tout au tout technique et nomenclature. Nous voudrions, par rapport à la langue et au sens commun, situer le vocabulaire de ces métiers, dont les praticiens sont très proches de la vie quotidienne. Aujourd' hui la langue des anciens métiers du bois abandonne peu à peu la langue commune, juste après le moment où elle s'était constituée en langue de spécialité bien répertoriée.
\end{abstract}

\begin{abstract}
Thanks to the quality and the continuity of the documentation available for France, the evolution of the vocabulary of woodworking can be traced from the 17th to the 20th century, when the more and more exclusive use of machinery completely modified both technique and nomenclature. Our aim is to situate the vocabulary of these trades, whose practioners are very close to day-to-day life, with relation to common language and common sense. Today, the language of the former woodworking trades is disappearing from the general lexicon, just after it had been made into a well-described special language.
\end{abstract}

Nous voudrions, dans cet article, exposer quelques réflexions sur la place d'une langue de métier en face de la langue commune, pour être plus précis, de celle de la langue «menuisière» dans la langue française. Il faut tout de suite faire observer qu'on peut envisager les langues de métiers sous trois aspects:

- la langue des ateliers ou argot de métier, qui caractérise la pratique orale entre gens de la même corporation. «Travailler en perruque», par exemple, c'est travailler pour son compte, à l'aide des outils, des machines, des fournitures du «singe» (le patron!).

- la littérature technique faite d'abord par les gens de métier, les ingénieurs, puis à partir de la fin du XIX ${ }^{\mathrm{e}}$ siècle, par les enseignants du technique. Il s'agit donc d'une littérature technologique et didactique.

- le lexique spécifique des techniques: outils (varlope), machines (toupie), matériaux (ronce de noyer), techniques opératives (corroyer). C'est la place de ces lexiques que nous examinerons ici.

Nous nous occuperons d'un Métier de Tradition: le travail du bois, menuiserie et ébénisterie principalement. C'est à partir du XVII ${ }^{\mathrm{e}}$ siècle, qu'apparaissent, en langue française, les premiers livres de métier traitant du travail du bois. Le premier est le Théâtre de l'Art de Charpenterie de Mathurin Jousse (trois éditions: 1627, 1642, 1702). Dans Des Principes de l'Architecture, de la Sculpture, de la Peinture et des Arts qui en dépendent (quatre éditions: 1676, 1690, 1697, 1776), André Félibien consacre le chapitre XIV à la charpenterie, le XVIII à la menuiserie, le XIX à la menuiserie de placage (édition 
de 1697). Dans l'Encyclopédie, c'est dans le volume VII des Planches (86 planches) et dans le volume X de Texte (p. 346-357) que 1'on traite de l'Art de la Menuiserie. C'est enfin André-Jacob Roubo qui fait paraître son Art du Menuisier (de 1770 à 1782), certainement un des plus beaux livres de la Description des Arts et Métiers, de l'Académie des Sciences. Le lexique technique, celui de l'ouvrage «fait main» en tout cas, n'acquerra pas beaucoup de termes nouveaux depuis Roubo, perfection et tradition se conjuguant pour ne pas susciter de désir d'innovation. Ce n'est qu'avec la généralisation des machines et des matériaux nouveaux (bois agglomérés ou panneaux de fibres par exemple) que la menuiserie, au $\mathrm{XX}^{\mathrm{e}}$ siècle, change de techniques, de dimension (industries du meuble) et donc de vocabulaire (illustration: la défonceuse, machine portable munie de mèches et de fraises, servant à rainurer et moulurer, qui remplace les bouvets et outils à moulures poussés à la main).

Mais ce n'est pas en historien des techniques que nous voulons aborder le domaine considéré, mais en linguiste.

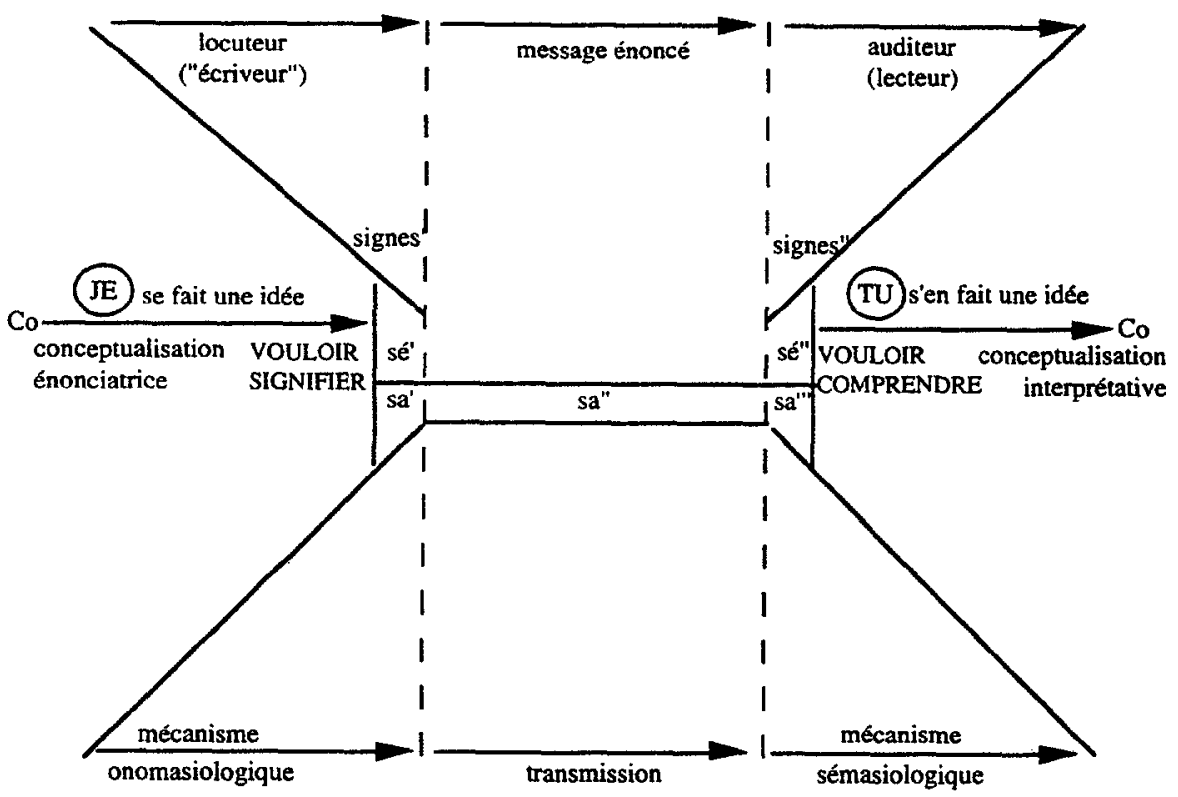

Ce schéma de la communication linguistique présente, de manière bien classique, les trois instances de l'acte de langage: émetteur (locuteur ou «écriveur»), message (séquence de sons ou suite de lettres), récepteur (auditeur ou lecteur).

Nous ne discuterons pas ici de certains problèmes (majeurs, il est vrai), qui ne sont pas ici de propos: symétrie en miroir des parcours onomasiologique et sémasiologique, à laquelle nous ne croyons guère, origine et nature de la conceptualisation qui génère l'énonciation...

Nous croyons que le langage intérieur, qu'il soit ou non prélude à message, de même que l'interprétation de ce message, se font EN SIGNES et SUR SIGNES. Le seul FAIT de langage, matériel, perceptible, enregistrable, mesurable (mais à quoi bon, sans doute !) est le signifiant. 
Nous voulons seulement montrer ici que tout énoncé linguistique et ses constituants de même nature significative, ressortissent de trois structurations :

1. une structuration en signes, FORMES phoniques (ou graphiques) dotées de SENS. Ce sont des signes bifaces : signifiant et signifié.

2. une structuration en signifiants, suites phoniques ou graphiques, articulées ou tracées, constituant des mots formels séparés par des pauses ou des blancs et qui sont la manifestation perceptible du caractère DISCRET des signes.

3. une structuration sémantique ou plutôt une matière sémantique, fluide et mouvante, c'est-à-dire continue, non discrète, preuves en sont anaphores et cataphores, métonymies, métaphores, évolutions sémantiques, etc.

Il nous semble que les trois types d'écritures confirment cette triple structuration:

- la pictographie qui est une stylisation des référents visualisables.

- la «phonographie» qui est une notation consonantique, syllabique, ou alphabétique des signifiants.

- l'idéographie qui, sous la forme de hiéroglyphes ou de caractères, est un système de symboles qui notent des signes, très analogues à 5 ou $\therefore$ (franc-maçon).

Il ne peut y avoir d'écriture du signifié ou du conceptuel (même les «schémas catastrophiques» de René Thom ont un signifiant graphique) et cela prouve, par défaut, l'hétérogénéité de la structuration du signifié par rapport aux deux autres.

Quoi qu'il en soit, l'articulation en signes (et signifiants) linéairement disposés font que ces éléments se définissent en syntagme (combinaisons ou virtualités combinatoires, contrastes et relations en présence) et en paradigme (commutations, choix d'oppositions, rapports en absence). C'est ce problème linguistique qui nous retient aujourd'hui : nous pensons que définir les rapports de la langue commune et de la langue des Arts et des Sciences nous oblige à élaborer une typologie des paradigmes.

A. La première typologie consiste à opposer deux macro-paradigmes : grammaire et lexique. Nous avons tenté d'en définir les dimensions dans l'Hommage à Bernard Pottier (1988, T. I, p. 268). En résumé, et en insistant sur la perméabilité des deux inventaires :

\begin{tabular}{|c|c|c|}
\hline & GRAMMAIRE & LEXIQUE \\
\hline $\begin{array}{l}\text { SIGNIFIANT } \\
\text { (langues plurisyllabiques) }\end{array}$ & $\begin{array}{l}\text { léger } \\
\text { atone }\end{array}$ & lourd \\
\hline en SYNTAGME & $\begin{array}{l}\text { combinabilité large } \\
\text { marginal }\end{array}$ & $\begin{array}{l}\text { combinabilité restreinte } \\
\text { central }\end{array}$ \\
\hline en PARADIGME & $\begin{array}{l}\text { fermé \& stable } \\
\text { monoparadigmatique }\end{array}$ & $\begin{array}{l}\text { ouvert } \& \text { changeant } \\
\text { polyparadigmatique }\end{array}$ \\
\hline SIGNIFIÉ & $\begin{array}{l}\text { léger } \\
\text { abstrait }\end{array}$ & $\begin{array}{l}\text { lourd } \\
\text { concret }\end{array}$ \\
\hline
\end{tabular}

B. Si l'on considère, d'un point de vue sociolinguistique, la répartition des utilisateurs, une deuxième typologie s'esquisse : 


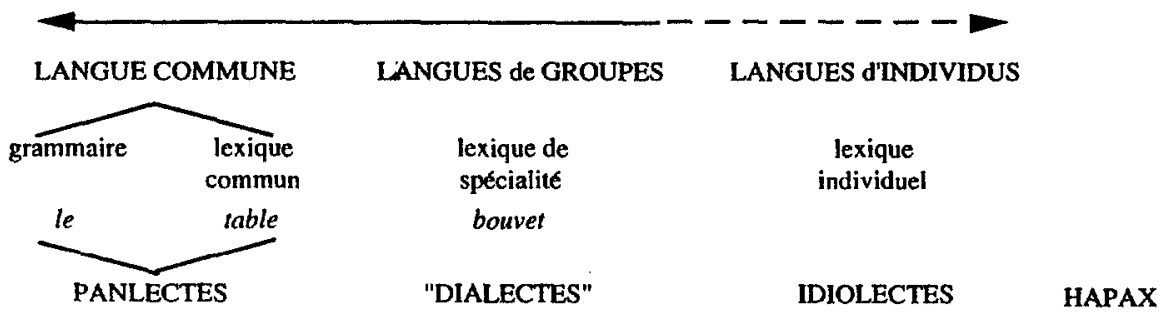

Mais il faut bien remarquer que les différences de groupes (métiers, classes sociales, associations sportives ou festives, familles, etc.) ou individuelles, se font toujours dans le cadre de la langue commune et qu'elles n'engendrent que des «diglossies» partielles et passagères.

C. Langue commune et langues des Arts et Métiers traditionnels s'opposent aux terminologies modernes par leur processus de formation:
tradition linguistique
tradition linguistique \& «oeuvrière»
$\longrightarrow$ langue commune
$\longrightarrow$ langue des Arts
$\longrightarrow$ terminologie
création volontaire à des fins opératoires

Il nous semble que l'intention terminologique est triple:

motivation: affirmation d'un savoir dans un lexique spécifique

- normalisation: création d'une norme et constitution d'un ensemble de termes,
pourvu, si possible, d'une régularité: sulfate, sulfite, sulfure ; pourvu, si possible, d'une régularité : sulfate, sulfite,

monosémie: les termes se doivent d'être univoques, de ne désigner qu'un unique référent.

Cette parenté entre langue commune et langue des Arts, explique les passages insensibles de l'une à l'autre: rabot appartient tout autant à la langue de tous qu'à la langue des artisans du bois (mais a-t-il le même sens?). Les Académiciens français s'en sont beaucoup préoccupés aux XVII et XVIII ${ }^{e}$ siècles et l'on constate, par exemple, que la quatrième édition du Dictionnaire de l'Académie (1762), ajoute seize noms d'outils aux quarante-huit que comptait la troisième (1740), dont la varlope, l'outil emblématique des Compagnons Menuisiers.

D. Par ailleurs, on peut observer que terminologie et langage des Arts s'opposent à la langue commune, en ce sens qu'ils ont un lien beaucoup plus intime avec le référent en raison de leur monosémisme. Pour un menuisier, un riflard est un riflard, en fonction de caractéristiques qui sont prégnantes aux yeux de l'artisan: c'est un long rabot à poignées, plus léger que la varlope, dont le fer est affûté légèrement arrondi, afin de pouvoir dégrossir sans trop d'effort les planches brutes de sciage. L'autre riflard, du nom de $\mathrm{M}$. Riflard, personnage de comédie, par antonomase, appartient à un autre micro-paradigme (parapluie / pépin/riflard) et n'est qu'un homonyme. 
E. Les lexiques des Arts ont cependant, nous semble-t-il, certaines particularités, où l'on aperçoit la relation entre dénomination et pensée opérative, le Geste et la Parole :

- beaucoup de déverbaux qui sont des noms d'action: lime, pince, plane, presse, râpe, scie, et rabot selon nous (in Actes du XVIII Congrès de Linguistique et Philologie Romanes, Trèves, 1986, T. IV, p. 459-466).

des noms d'agent: feuilleret, racloir, tenaille.

des métaphores imagées: le bouvet, c'est un petit bœuf qui creuse son sillon, sa rainure.

des composés et des périphrases motivées : serre-joints, rabot à dégrossir.

Une source de formation est le passage des hyperonymes (qui le sont aussi en langue commune) à une sorte de préfixes classificateurs : ciseau, gouge, rabot, scie... :

$\begin{array}{lll}\text { en vue d'une action } & \text { adaptée à un objet } & \text { forme } \\ \text { scie à araser } & \text { scie à tenons } & \text { scie à cadre } \\ \text { scie à refendre } & \text { scie à placage } & \text { scie à dos } \\ \text { scie à débiter } & \text { scie à cheville } & \text { scie égö̈ne }\end{array}$

Nos modestes réflexions sur une typologie des paradigmes nous ont rendu quelque peu sceptique vis-à-vis des macro-paradigmes, dont le Thesaurus est l'exemple «hénaurme». Ces sortes de paradigmes existent-ils en dehors de l'imagination des linguistes et des lexicographes?

Les significations se déterminent par rapport à des micro-paradigmes en jeu dans les circonstances particulières des discours :

$\mathrm{JE} / v s / \mathrm{TU}$ ou JE/vs/VOUS, suivant notre interlocuteur; TABLE/vs/BUREAU, ou TABLE/vs/BIGORNE (enclume du forgeron). À cela il faut ajouter que les éléments du lexique des métiers s'intègrent dans des micro-ensembles dont l'organisation est en partie liée aux nécessités opératives et ne font donc pas partie de simples nomenclatures: l'affútage, c'est le micro-paradigme, rabot, riflard, varlope, et c'est l'ensemble des outils qui servent à corroyer le bois; et la varlope ne se conçoit pas sans l'établi qui supporte la planche à replanir et à tirer d'épaisseur, et sans la griffe fixée dans cet établi et qui sert à maintenir l'ouvrage. 